\title{
An Interference Analysis Method with Site-Specific Path Loss Model for Wireless Personal Area Network
}

\author{
Hyun Wook Moon ${ }^{1} \cdot$ Sewoong Kwon ${ }^{2} \cdot$ Jong Hyun Lee ${ }^{2} \cdot$ Young Joong Yoon ${ }^{1}$
}

\begin{abstract}
In this paper, an interference analysis method with a site-specific path loss model for a wireless personal area network (WPAN) is proposed. The site-specific path loss model is based on geometrical optics and geometric probability to consider both site-specific radio propagation characteristics and a closed-form expression to obtain the mean interference from which the uniformly distributed multiple interferers are derived. Therefore, the proposed interference analysis method can achieve more computational simplicity than the Monte-Carlo (MC) simulation, which uses the ray-tracing (RT) technique. In addition, better accuracy than the conventional interference analysis model that uses stochastic method can also be achieved. To evaluate the proposed method, a signal to the interference-noise ratio with a mean interference concept for uniformly distributed interferers is calculated and compared in two simulation scenarios. As a result, the proposed method produces not only better matched results with the MC simulation using the RT technique than the conventional interference analysis model, but also simpler and faster calculation, which is due to the site-specific path loss model and closed-form expression for interference calculation.
\end{abstract}

Key words : Interference Analysis, Monte-Carlo (MC), Path Loss, Wireless Personal Area Network (WPAN).

\section{Introduction}

As the demand for wireless communication services for indoor environments to provide multi-function and high data rate increase, more and more electrical devices short-range radio interfaces such as ultra-wideband (UWB), Zigbee, and Bluetooth are developed. These wireless communication technologies are standardized in the IEEE 802. 15 task group for wireless personal area networks (WPAN) [1]. In addition, a wireless sensor network is composed by these applications for each service target. However, because these applications generally utilize common unlicensed industrial, scientific, and medical (ISM) bands, they have difficulty in controlling the co-

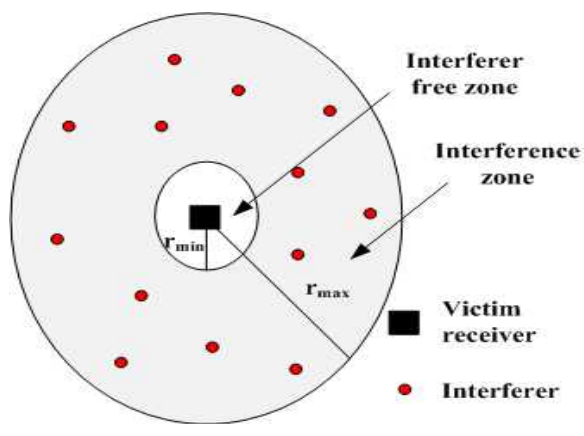

Fig. 1. Geometry of interference model. mmunication services for multi-users. Therefore, these applications can interfere with each other because they tend to be located in close proximity. In addition, UWB techniques, which are considered a means of supporting a high data rate, reuse the existing radio spectrum so that UWB systems can generate interference with other radio communication systems. For these reasons, many studies on the coexistence of different communication systems in WPAN applications or wireless local area network (WLAN) applications have been done [2 4].

The methods of analyzing interference effects are categorized into minimum coupling loss (MCL), enhanced MCL (E-MCL), and the Monte-Carlo (MC) based method. The E-MCL method is also known as the stochastic method; it assumes that interferers are uniformly distributed in an interference zone as shown in Fig. 1. In this method, the path loss is calculated by the simple and empirical path loss model with path loss exponents for indoor environments. Conventional interference analysis is performed mainly by this stochastic method. Although this method can provide a fast and closed-form solution for interference calculation, it can not consider site-specific radio propagation characteristics [5]. On the other hand, the MC method is a statistical technique that can address virtually all radio-interference scenarios. In this paper, the MC method using the RT

Manuscript received October 1, 2010 ; revised December 3, 2010. (ID No. 20101001-030J)

${ }^{1}$ School of Electrical \& Electronic Engineering, Yonsei University, Seoul, Korea.

${ }^{2}$ LIG Nex1 Corporation, Yongin, Korea.

Corresponding Author : Young Joong Yoo (e-mail : yjyoon@yonsei.ac.kr) 
technique is employed as a reference result and compared because RT techniques allow exact site-specific propagation characteristics. However, the MC method using RT techniques needs a very large number of calculations [6]. Therefore, an interference analysis method with a site-specific path loss model based on geometrical optics and geometric probability is proposed. This proposed method can achieve improved computational simplicity over the MC method that uses RT techniques and better accuracy than the conventional interference analysis model that uses the stochastic method.

This paper is organized as follows. In Section $\Pi$, the site-specific path loss model and the closed-form expressions for the interference calculation of the proposed method are presented. In Section III, the simulation results of the proposed method are compared and validated. Finally, concluding remarks are made in Section IV.

\section{II . A Proposed Interference Analysis Method}

In this section, an interference analysis method with a site-specific path loss model based on geometric optics and geometric probability is proposed.

\section{2-1 Site-Specific Path Loss Model based on Geometric Optics and Geometric Probability [7]}

When the transmitting power from transmitter (Tx) is radiated and arrived at a receiving point $(\mathrm{Rx})$, the propagating rays experience many reflections and transmissions due to object-intersections. Consequently, the path power tends to decay rapidly with distance at a rate more than the inverse-square distance law for the free- space. In other words, each propagating ray loses a certain amount of power due to intersection loss from reflection or transmission. On the other hands, other propagation mechanisms such as diffraction and scattering can be ignored in indoor propagation [8]. This intersection loss can be expressed in a power formulation as a multiplication by a loss coefficient. Therefore, each ray path power is expressed by Eq. (1) where ray propagates $l$ meters from the Tx with $n$ intersections ( $m$ reflections and $(n-m)$ transmissions).

$$
P(l, n, m)=P_{0} l^{-2} R^{2 m} T^{2(n-m)}
$$

where $R$ and $T$ are the reflection and transmission coefficients, respectively. $P_{o}$ is the free-space power at distance of 1 meter for omni-directional antenna. Using the probability density function (PDF), $f(n, m \mid l)$, of a path that intersects $n$ objects after traveling distance $l$ with $m$ reflections and $(n-m)$ transmissions, the mean path power can be expressed in Eq. (2) as follows:

$$
P(l)=P_{0} l^{-2} \sum_{n=0}^{\infty} \sum_{m=0}^{n} f(n, m \mid l) R^{2 m} T^{2(n-m)}
$$

The process of hitting $n$ obstacles can be considered to be a combination of reflections and transmissions. In addition, these two events are independent and exclusive in one path at one instance. Therefore, $f(n, m \mid l)$ can be decomposed as a multiplication of two PDFs as in Eq. (3):

$$
f(n, m \mid l)=f_{1}(n, l) f_{2}(m \mid n, l)
$$

where $f_{1}(n \mid l)$ is the PDF in which a ray undergoes $n$ intersections for traveling distance $l$. This PDF can be represented by the Poisson distribution for indoor environment [9]:

$$
f_{1}(n \mid l)=\frac{(\beta l)^{n}}{n !} e^{-\beta l}
$$

where $1 / \beta$ is the mean free distance between two intersections. This parameter is defined as the mean distance between two intersections and estimated according to the given geometry. In addition, $f_{2}(m \mid n, l)$ is the PDF of the ray with $m$ reflections and $(n-m)$ transmissions. Since these are independent and exclusive, a binomial PDF fits these conditions [10]. Then

$$
f_{2}(m \mid n, l)=\left(\begin{array}{c}
n \\
m
\end{array}\right) p^{m}(l) q^{(n-m)}(l)
$$

where $p(l)$ and $q(l)$ are the probabilities of reflection and transmission, respectively. Substituting (4) and (5) in (2) yields

$$
P(l)=P_{0} l^{-2} e^{-\beta l} e^{\beta l\left(q T^{2}+p R^{2}\right)}
$$

At this moment, $p(l)$ and $q(l)$ can be modeled as follows:

$$
\begin{aligned}
& p(l)=\frac{1-e^{-\beta \Delta}}{2} \\
& q(l)=\frac{1+e^{-\beta \Delta}}{2}
\end{aligned}
$$

where $\Delta$ is the difference between the Tx-Rx distance $(L)$ and total path length $(l)$. This model is demonstrated by a Monte Carlo simulation [7]. Thus, substituting (7) and (8) in (6), the final path loss equation for the multi-path is determined as below.

$$
P(l)=P_{0} l^{-2} e^{-\beta l} e^{\frac{\beta l\left(T^{2}+R^{2}\right)}{2}} e^{\frac{\beta l\left(T^{2}-R^{2}\right) e^{-\Delta \beta}}{2}}
$$

and the total power received from multi-path at Tx-Rx distance $L$ can be obtained by summation of each multi-path power without phase [11]. 


$$
P_{t}(L)=\int_{l=L}^{\infty} P(l) d l \cong \int_{l=L}^{l_{\max }} P(l) d l
$$

In order to avoid complications, the total received power from multi-paths is expressed as $P_{t}$. In practice, the summation for all multi-paths is impossible, so the integral is calculated in the range from $L$ to $l_{\max }$ and $l_{\max }$ is the maximum path length and computed as in Eq. (11).

$$
P_{t h}=10 \log _{10}\left(\frac{P_{\max }}{P\left(l_{\max }\right)}\right)
$$

where $P_{\max }$ is the maximum power of a ray that travels from $\mathrm{Tx}$ to $\mathrm{Rx}$. For $P_{t h}=10 \mathrm{~dB}, P\left(l_{\max }\right)=0.1 P_{\max }$. From numerous evaluations, the $l_{\max }$ is determined by the following equations: $l_{\max }=3 L$ for LOS (Scenario A) and $l_{\max }=1.5 \mathrm{~L}$ for NLOS (Scenario B). Thus, in the above equation (10), the unknown parameter is $\beta$. This parameter represents the site-specific characteristics of the given geometry, and the values for the interference scenarios are presented in Section III.

\section{2-2 Interference Analysis Formula}

As mentioned earlier, Fig. 1 shows the geometry of the interference model. The victim receiver is placed at the center of two circles. The inner circle is defined by the boundary of an interference free zone. In other words, there are no interferers inside the interference free zone. The interferers are located between the inner and outer circles with uniform distribution.

$$
f(r)=\left\{\begin{array}{cl}
0 & r<r_{\min }, r>r_{\max } \\
\frac{2 r}{r_{\max }^{2}-r_{\min }^{2}} & r_{\min } \leq r \leq r_{\max }
\end{array}\right.
$$

For a single interferer, the mean interference can be obtained by the integration of interference power and distribution probability.

$$
I_{1}=\int_{r_{\min }}^{r_{\max }} P(r) f(r) d r=F\left(r_{\max }\right)-F\left(r_{\min }\right)
$$

where $F(\bullet)$ is the indefinite integral of right-hand side of (13). From (9) and (10), the indefinite integral $F(r)$ is derived by closed-form.

$$
\begin{aligned}
& F(r)=-\frac{1}{(l+r)\left(r_{\max }^{2}-r_{\min }^{2}\right)} e^{-\beta l} \times \\
& \left(\begin{array}{l}
2 e^{\frac{1}{2} e^{-\beta l}\left[(l+r)\left(R^{2}-T^{2}\right)+e^{\beta l}\left(L\left(-2+R^{2}+T^{2}\right)+l\left(R^{2}+T^{2}\right)\right)\right]} \\
-(l+r)\left[\left(R^{2}-T^{2}\right) \beta l+e^{\beta l}\left(\left(R^{2}+T^{2}-2\right) \beta l-2\right.\right.
\end{array}\right)
\end{aligned}
$$

If the mean density of interferers is $\rho$, the mean number of interferers in the region $r_{\min }<r<r_{\max }$ is calculated by multiplication of the mean density of the interferers and area of the interference zone.

$$
\bar{N}=\rho \pi\left(r_{\max }^{2}-r_{\min }^{2}\right)
$$

Finally, the mean interference of the victim receiver from multiple interferers can be obtained.

$$
\bar{I}=\bar{N} \cdot I_{1}=\rho \pi\left(r_{\max }^{2}-r_{\min }^{2}\right) I_{1}
$$

\section{Simulation Results}

To evaluate the performance of the proposed method, the proposed site-specific interference analysis method is compared with the conventional interference analysis model with the stochastic method and the MC method using the RT technique.

\section{3-1 Simulation Scenarios and Site-Specific Parameters}

In this paper, two simulation scenarios for indoor environments are considered. Scenario A is a large room in which many interferers are located in the LOS region and the interference effect is more critical. On the other hands, scenario B is a small room in which almost all interferers are located in the NLOS region. Hence, the interference effect is smaller because the interference from the NLOS region has more attenuation due to various objects. Furthermore, the desired signal has also more attenuation along the Tx-Rx distance. Fig. 2 and Fig. 3 show the simulation scenarios, and the size of both rooms is described in Table 1 .

To calculate the received power and interference using the path loss model which is described in Section $\Pi$, the mean free distance for each simulation scenario is necessary. This parameter for each room is computed by geometrical probability with the rectangular shape and total mean free distance can be obtained by averaging over the interference zone area of weighted sum of individual room [12]. Hence, the total mean free distance for each simulation scenario is as follows, respectively.

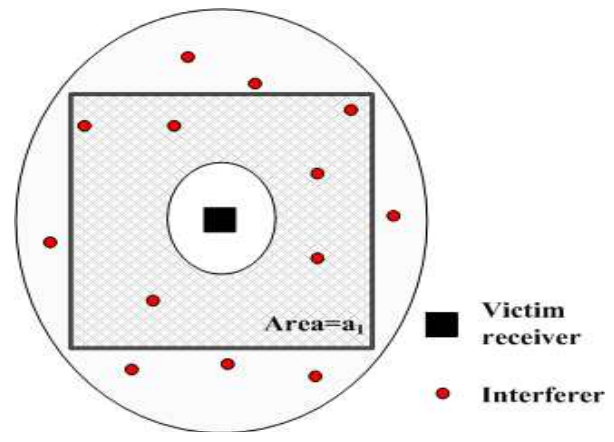

Fig. 2. Scenario A (large-size room). 


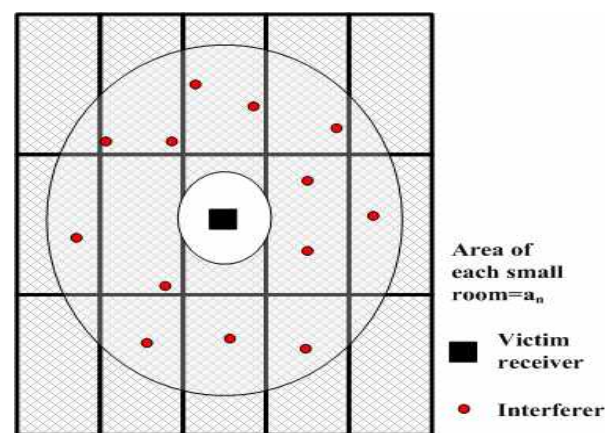

Fig. 3. Scenario B (small-size room).

Table 1. Parameters of simulation environments.

\begin{tabular}{|l|c|c|c|c|}
\hline & $\begin{array}{c}\text { Width } \\
{[\mathrm{m}]}\end{array}$ & $\begin{array}{c}\text { Length } \\
{[\mathrm{m}]}\end{array}$ & $\begin{array}{c}\text { Height } \\
{[\mathrm{m}]}\end{array}$ & $1 / \beta$ \\
\hline Scenario A & 8.4 & 8.5 & 2.62 & 3.23 \\
\hline Scenario B & 4 & 6 & 2.62 & 2.5 \\
\hline
\end{tabular}

Scenario A:

$\frac{1}{\beta}=\frac{a_{1}}{\pi r^{2}} \cdot \frac{1}{\beta_{1}}$

Scenario B:

$\frac{1}{\beta}=\sum_{n=1}^{N} \frac{a_{n}}{\pi r^{2}} \cdot \frac{1}{\beta_{n}}$

where $a_{1}$ and $a_{n}$ are the areas of the single rooms in scenario $\mathrm{A}$ and scenario $\mathrm{B}$, respectively. $1 / \beta_{1}$ and $1 / \beta_{n}$ are the mean free distance for the single rooms in scenario A and scenario B, respectively. $r$ indicates the $r_{\max }$ in Fig. 1. Thus, the total mean free distances for scenario A and $\mathrm{B}$ are obtained in Table 1 .

\section{3-2 Validation of the Proposed Method}

In order to validate the proposed method, the mean signal to the interference-noise ratio (SINR) according to the Tx-Rx distance is calculated and compared with the conventional interference analysis model with stochastic method and MC method using RT technique. The path loss exponent of the conventional interference analysis model is based on the IEEE 802.15.4a channel model [13]. In addition, to perform the MC simulation using RT and simulate in identical conditions, the 5,000 interferers are located in an interference zone with uniform distribution. Fig. 4 and Fig. 5 show the comparison of the SINR results for scenario A and scenario $\mathrm{B}$, respectively. In these figures, the conventional interference model with the stochastic method is indicated by 'stochastic model.' As shown in Fig. 4 and Fig. 5, the results of the proposed method are in good agreement with the MC simulation using RT. The root mean square (RMS) errors between the proposed method or the conventional model and the MC method are listed in Table 2. As a result, the RMS error of the proposed method is smaller than that of the conventional interference analysis model at 5.76 $\mathrm{dB}$ for scenario $\mathrm{A}$ and $3.99 \mathrm{~dB}$ for scenario $\mathrm{B}$, respectively. In addition, the results of the conventional interference model, i.e., the 'stochastic model', are the same for both scenarios because they can not reflect real environments. Thus, these results show that the proposed meth-

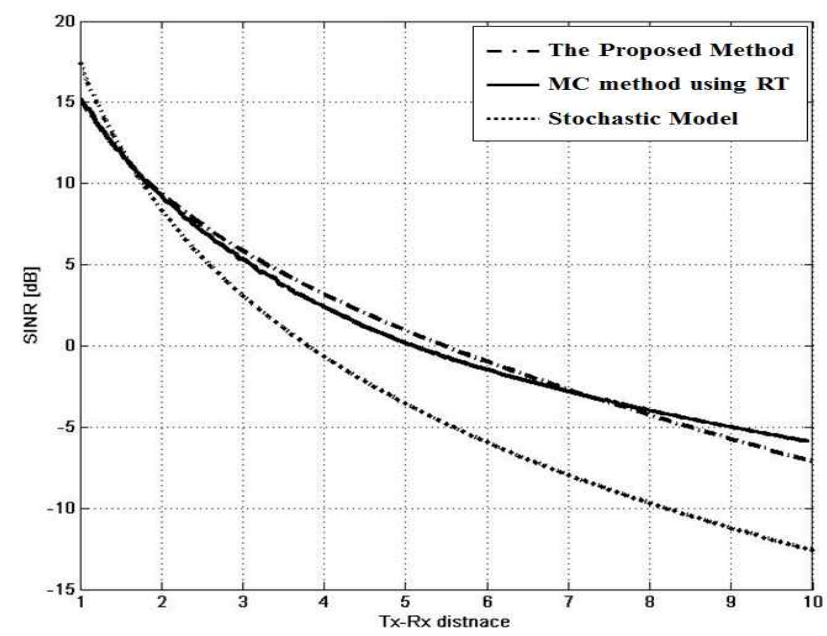

Fig. 4. Comparison of SINR results (Scenario A).

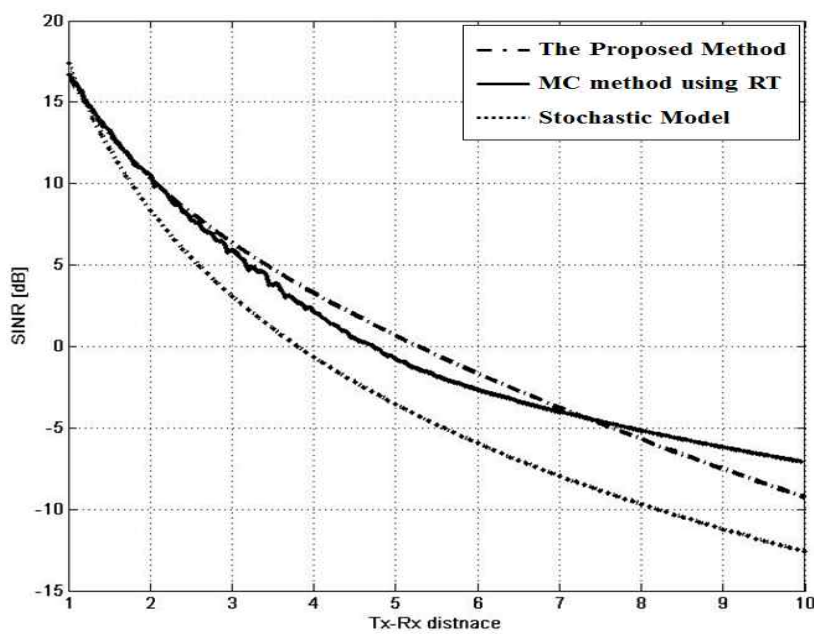

Fig. 5. Comparison of SINR results (Scenario B).

Table 2. RMS error with MC method using RT techniques.

\begin{tabular}{|l|c|c|}
\hline & $\begin{array}{c}\text { The proposed } \\
\text { method }\end{array}$ & $\begin{array}{c}\text { Conventional } \\
\text { interference analysis } \\
\text { model with stochastic } \\
\text { method }\end{array}$ \\
\hline Scenario A & $0.47 \mathrm{~dB}$ & $6.23 \mathrm{~dB}$ \\
\hline Scenario B & $1.34 \mathrm{~dB}$ & $5.33 \mathrm{~dB}$ \\
\hline
\end{tabular}


od can reflect the site-specific characteristics. However, the proposed method underestimates the SINR for short range and overestimates the SINR as the Tx-Rx distance increases since the calculated desired signal level is low for the underestimation region and high for the overestimation region. In addition, the error of scenario $\mathrm{B}$ for the proposed method is larger than that of scenario A, which means that the mean free distance calculation is inaccurate for complex environments. However, scenario B is the most complex indoor environment, and the RMS error for scenario B is about $1.34 \mathrm{~dB}$. Thus, the proposed method is suitable for SINR calculation for WPAN applications.

In addition, the calculation of the proposed method is simple and quick. For RT with ray shooting, the RT tool starts by shooting $(M)$ rays from the transmitter in all directions. If there are $N$ wall intersections, and each ray splits into two "child" rays- a reflected ray and a transmitted ray- the number of operations in RT is proportional to $O\left(M \cdot\left(2^{N+1}-1\right) \cdot N\right)$ [14], and the post- processing for the MC simulation using the calculated received powers is required. However, the received power in the proposed method is simply calculated using Eq. (9) and (10). The mean interference power can be obtained from closed form expression, Eqs. (13) and (14). Therefore, the proposed method has more accurate results than the conventional interference analysis model and can calculates the SINR in a simple and quick manner.

\section{Conclusion}

In this paper, the interference analysis method with the site-specific path loss model for WPAN is proposed. This proposed method not only provides better accuracy than the conventional interference analysis model with the stochastic method but also results in more efficiency than the MC method using RT computationally due to closed-form expression. Although the RMS error of the proposed method increases in more complex environments, low RMS errors for both scenarios exhibit that the proposed method can reflect site-specific characteristics. In addition, the interference calculation is not dependent on system parameters such as transmitting power and, the modulation scheme because the interference is calculated from only path loss concept. Thus, by adjusting the system parameters on the calculated interference, as in previous interference analysis studies, a more accurate interference analysis can be conducted.

The authors are gratefully acknowledging the financial support by Defense Acquisition Program Administration and Agency for Defense Development under the contract UD100002KD.

\section{References}

[1] J. Karaoguz, "High-rate wireless personal area networks," IEEE Commun. Mag., vol. 39, no. 12, pp. 96-102, Dec. 2001.

[2] D. Landi, C. Fischer, "The effects of UWB interference on GSM systems," in Proc. of Int. Zurich Seminar, pp. 86-89, Feb. 2004.

[3] "Study of interference effects of a UWB mass deployment on GSM systems," ITU Document, 1-8/9E, Oct. 2003.

[4] "UWB interference with Bluetooth," ITU Document 1-8/11-E, Oct. 2003.

[5] "A comparison of the minimum coupling loss method, enhanced minimum coupling loss method, and the monte-carlo simulation," ERC Report 101, May 1999.

[6] "Monte-Carlo simulation methodology for the use in sharing and compatibility studies between different radio service or system," ERC Report 68, Jun. 2002.

[7] M. Hassan-Ali, K. Pahlavan, "A new statistical model for site-specific indoor radio propagation prediction based on geometric optics and geometric probability," IEEE Trans. Wireless Commun., vol. 1, no. 1, pp. 112-124, Jan. 2002.

[8] H. Bertoni, W. Honcharenko, L. R. Maciel, and H. $\mathrm{Xia}$, "UHF propagation prediction for wireless personal communications," Proc. IEEE, vol. 82, pp. 13331359, Sep. 1994.

[9] M. Hassan-Ali, "Using ray-tracing techniques in sitespecific statistical modeling of indoor radio channels," Ph. D. dissertation, Worcester Polytechnic Institute, Worcester, MA, 1998.

[10] A. Svenshnikov, Probelms in Probability Theory, Mathematical Statistics and Theory of Random Functions, New York: Dover, 1968.

[11] R. Valenzuela, O. Landron, and D. Jacob, "Estimating local mean signal strength of indoor multipath propagation," IEEE Trans. Veh. Technol., vol. 46, pp. 203-212, Feb. 1997.

[12] L. Santalo, Integral Geometry and Geometric Probability. Reading, MA: Addison-Wesly, 1976

[13] A. F. Molisch et al., "A comprehensive standardized model for ultrawideband propagation channels," IEEE TRans. Antennas Propag., vol. 54, no. 11, pp. 3151-3166, Nov. 2006.

[14] S. Fortune, "A beam-tracing algorithm for prediction of inddo radio propagation," in Porc. First ACM Workshop on Applied Computational Geometry, pp. 76-81, 1996. 


\section{Hyun Wook Moon}

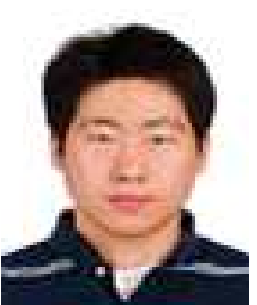

He received the B.S. and M.S. degree in electrical \& electronic engineering from Yonsei Univeristy, Seoul, Korea, in 2005 and 2007, respectively. $\mathrm{He}$ is currently working toward in the Ph.D. degree in the School of Electrical \& Electronic Engineering, Yonsei University. His research interests are in the areas of antenna and wave propagation

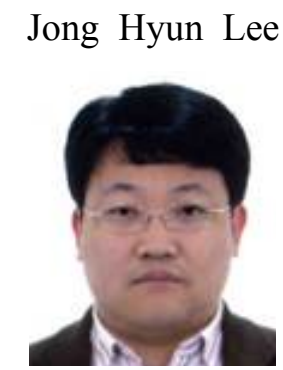

He received the B.S. degrees in electronic engineering from Pusan National University, Pusan, Korea, in 1997, and the M.S. and $\mathrm{Ph} . \mathrm{D}$. degree in electrical engineering from the Korea Advanced Institute of Science and Technology (KAIST) in 1999, and 2005 respectively. Since 2005 he has been working for research engineer at LIG Nex1. His main interests are signal processing, radar and communication channel modeling, and radar system design.

\section{Young Joong Yoon}

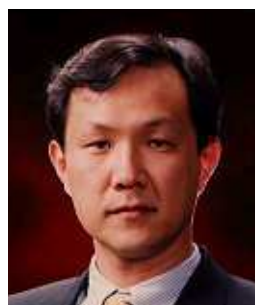

He received the B.S. and M.S. degrees in electronic engineering from Yonsei University, Seoul, Korea, in 1981 and 1986, respectively, and the Ph.D. degree in electrical engineering from the Georgia Institute of Technology, Atlanta, in 1991. From 1992 to 1993 , he was a Senior Researcher with the Electronics and Telecommunications Research Institute (ETRI), Daejeon, Korea. In 1993, he joined the faculty of Yonsei University, where he is currently a professor with the school of electrical and electronic engineering. Currently, he is a Vice President at the Korean Institute of Electromagnetic Engineering \& Science (KIEES). His research interests are antennas, RF devices, and radio propagations. 